\title{
Methylene Blue Dye-Induced Skin Necrosis in Immediate Breast Reconstruction: Evaluation and Management
}

\author{
Ji Hwan Lee ${ }^{1}$, Choong Hyun Chang ${ }^{1}$, Chan Heun Park ${ }^{2}$, June-Kyu Kim ${ }^{1}$ \\ Departments of ${ }^{1}$ Plastic and Reconstructive Surgery and ${ }^{2}$ Breast and Thyroid Cancer, Kangbuk Samsung Hospital, Sungkyunkwan University \\ School of Medicine, Seoul, Korea
}

Background For early breast cancer patients, skin-sparing mastectomy or nipple-sparing mastectomy with sentinel lymph node biopsy has become the mainstream treatment for immediate breast reconstruction in possible cases. However, a few cases of skin necrosis caused by methylene blue dye (MBD) used for sentinel lymph node localization have been reported.

Methods Immediate breast reconstruction using a silicone implant was performed on 35 breasts of 34 patients after mastectomy. For sentinel lymph node localization, 1\% MBD $(3 \mathrm{~mL})$ was injected into the subareolar area. The operation site was inspected in the postoperative evaluation.

Results Six cases of immediate breast reconstruction using implants were complicated by methylene blue dye. One case of local infection was improved by conservative treatment. In two cases, partial necrosis and wound dehiscence of the incision areas were observed; thus, debridement and closure were performed. Of the three cases of wide skin necrosis, two cases underwent removal of the dead tissue and implants, followed by primary closure. In the other case, the breast implant was salvaged using latissimus dorsi musculocutaneous flap reconstruction.

Conclusions The complications were caused by MBD toxicity, which aggravated blood disturbance and skin tension after implant insertion. When planning immediate breast reconstruction using silicone implants, complications of MBD should be discussed in detail prior to surgery, and appropriate management in the event of complications is required.

Keywords Breast implantation / Necrosis / Methylene blue / Sentinel lymph node biopsy / Breast neoplasm

\author{
Correspondence: June-Kyu Kim \\ Department of Plastic and \\ Reconstructive Surgery, Kangbuk \\ Samsung Hospital, Sungkyunkwan \\ University School of Medicine, 29 \\ Saemunan-ro, Jongno-gu, Seoul \\ 110-746, Korea \\ Tel: $+82-2-2001-2178$ \\ Fax: +82-2-2001-2177 \\ E-mail: Crossmatching@hanmail.net
}

No potential conflict of interest relevant to this article was reported.

Received: 22 Jul 2013 • Revised: 22 Aug 2013 • Accepted: 9 Sep 2013

pISSN: 2234-6163 • elSSN: 2234-6171・ http://dx.doi.org/10.5999/aps.2014.41.3.258・ Arch Plast Surg 2014;41:258-263

\section{INTRODUCTION}

In early breast cancer, such as ductal cell carcinoma in situ (DCIS) and all clinically node-negative T1-T3 invasive breast cancers, sentinel lymph node biopsy (SLNB) is performed [1]. SLNB is advantageous in that it allows one to avoid unnecessary axillary lymph node dissections and reduces complications associated with axillary lymph node dissection while sparing the

Copyright $(\odot 2014$ The Korean Society of Plastic and Reconstructive Surgeons

This is an Open Access article distributed under the terms of the Creative Commons Attribution Non-Commercial License (http://creativecommons.org/

licenses/by-nc/3.0/) which permits unrestricted non-commercial use, distribution, and reproduction in any medium, provided the original work is properly cited. 
volume of the axillary lymph node [2]. This procedure offers both aesthetic and clinical benefits for breast reconstruction.

Localization of the sentinel lymph node is a relatively accurate method of predicting the state of the axillary lymph node using isosulfan blue dye (IBD) or methylene blue dye (MBD). Recently, MBD has replaced IBD. Compared to IBD, MBD has a similar success rate of localization; nevertheless, it has a lower incidence of complications than IBD [3].

In early breast cancer, skin-sparing mastectomy or nipplesparing mastectomy is considered a safe oncologic method and is widely used for breast reconstruction [4]. Recently, nipplesparing mastectomy has been widely performed for DCIS, multicentric cancer, invasive breast cancer less than $5 \mathrm{~cm}$ in size, and prophylactic risk-reduction surgery. However, extensive carcinoma involvement in the skin, inflammatory breast cancer, Paget disease, and clinically suspicious nipple are absolute contraindications for nipple-sparing mastectomy [5].

In the case of preserving the nipple areolar complex (NAC), immediate breast reconstruction can be performed with a silicone implant with many aesthetic benefits [6]. Performing nipple-sparing mastectomy (or skin-sparing mastectomy) with simultaneous SLNB using MBD, we experienced more complications of MBD than in usual cases like modified radical mastectomy. Among the patients who underwent immediate breast reconstruction using silicone implants, we observed six cases of early complications associated with MBD.

Evaluation and appropriate handling of complications are discussed below. Furthermore, we reviewed and discussed MBD toxicity, aggravating factors, and their management.

\section{METHODS}

From February 2009 to March 2012, nipple-sparing mastectomy (or skin-sparing mastectomy) was performed on 35 breasts of 34 patients by two breast surgeons, followed by immediate breast reconstruction using silicone implants by one plastic and reconstructive surgeon (Table 1). Ten cases underwent skinsparing mastectomy due to positive nipple core biopsy. A retrospective chart review was performed, and the research protocol was approved by the local ethics committee.

\section{Sentinel lymph node biopsy and mastectomy}

For SLNB, 1\% MBD was injected into the areola skin. The total volume of MBD was approximately 3 to $5 \mathrm{~mL}$. Then, an incision was made near the axilla to obtain stained lymph nodes, and biopsy was performed. Through a lateral periareolar incision, the breast parenchyma was completely removed, leaving the skin envelope. In the case of nipple-sparing mastectomy, the nipple
Table 1. Patient characteristics

\begin{tabular}{|lc|}
\hline Characteristic & Total patients $(\mathbf{n = 3 4 )}$ \\
\hline Age $(\mathrm{yr})$ & 45.37 (range, 31-61) \\
BMI $\left(\mathrm{kg} / \mathrm{m}^{2}\right)$ & 22.26 (range, 17.32-29.39) \\
Underlying disease $(\mathrm{n})$ & Tuberculosis 1, hepatitis 1 \\
Implant volume $(\mathrm{mL})$ & 246 (range, $100-450)$ \\
Cases of using dermal matrix $(\mathrm{n})$ & 31 \\
Cases of NSM $(\mathrm{n}) / \mathrm{SSM}(\mathrm{n})$ & $25 / 10$ \\
Postoperative radiotherapy & None \\
\hline BMl, body mass index; NSM, nipple-sparing mastectomy; SSM, skin-sparing \\
mastectomy.
\end{tabular}

core was biopsied. If the result of frozen biopsy was free from cancer, the NAC was spared for breast reconstruction.

\section{Breast reconstruction}

After performing skin-sparing mastectomy or nipple-sparing mastectomy, breast reconstruction was initiated. First, the circulation of the skin envelope was checked. Then, the pectoralis major muscle was separated from the ribs. Next, the dissection of the pectoralis major muscle to the vicinity of the sternum and the retropectoral pocket was made. After a sizer was inserted into the pocket, we made a symmetric inframammary fold and determined the implant size. Finally, the state of the skin flap was checked, and additional debridement was performed for the parts suspected of necrosis. For all patients, the texturedtype silicone implant (Siltex implant, Mentor Corp., Santa Barbara, CA, USA) was inserted into the retropectoral pocket. In order to prevent direct contact from the lateral incision area and to cover the areas lateral to the muscle layer, acellular dermal matrix (AlloDerm, LifeCell Corp., Branchburg, NJ, USA), 4 $\mathrm{cm} \times 12 \mathrm{~cm}$ or $4 \mathrm{~cm} \times 16 \mathrm{~cm}$ in size was used. Two Jackson-Pratt drains were inserted to the pocket's upper and lower poles, followed by skin closure.

\section{Postoperative evaluation}

The color and temperature of the skin flap and the amount and color of drainage were observed daily and evaluated. Dressing changes were carried out every two days.

\section{Statistical analysis}

The $t$-test was used to compare the mean of continuous variables, and all tests were two sided. An analysis was carried out using SAS software (ver. 9.2). P-values $<0.05$ were considered statistically significant.

\section{RESULTS}

MBD staining was associated with skin complications in 6 cases 
of 35 mastectomies, 5 out of 25 nipple-sparing mastectomy patients, and 1 out of 10 skin-sparing mastectomy patients. Compared with non-complicated cases, there was no relationship between patient age and body mass index, and implant size (Table 2).

Among six complicated breasts, one case demonstrated localized blue staining and peripheral redness on the skin surface, but the conditions improved with conservative treatment. In two cases, skin necrosis at the incision area near the areola was observed. After 14 days, wound dehiscence or NAC necrosis was observed. Consequently, under local anesthesia, debridement was performed at the outpatient clinic.

In the other three cases, the size of the blue-stained area was over $5 \mathrm{~cm} \times 3 \mathrm{~cm}$, including NAC. In the first three days, sluggish capillary filling was observed in the blanching test. Moreover, the temperature of the skin surface was lower than that of the surrounding areas. After 3 to 5 days, bullae were observed on the stained skin surface. Following this, skin necrosis advanced on the stained areas with a distinct boundary. Because necrosis of the skin and subcutaneous tissue can cause further infection, excision of the necrotic tissue was planned. In one case, five days after surgery, the implant was preserved by using a pedicled latissimus dorsi musculocutaneous flap. In two cases, necrotic tissue and the silicone implants were removed on the sixth and the fourteenth day after the operation due to the patients' refusal to undergo additional surgery for breast reconstruction (Fig. 1).

Blue staining was observed in all layers of removed skin as

Table 2. Comparative analysis of complications

\begin{tabular}{|lccc|}
\hline Characteristic & $\begin{array}{c}\text { Non-complicated } \\
\text { cases }(\mathbf{n}=\mathbf{2 8})\end{array}$ & $\begin{array}{c}\text { Complicated } \\
\text { cases }(\mathbf{n}=\mathbf{6})\end{array}$ & P-value \\
\hline Age $(\mathrm{yr})$ & $43.68 \pm 7.24$ & $45.83 \pm 8.23$ & 0.49 \\
Body mass index $\left(\mathrm{kg} / \mathrm{m}^{2}\right)$ & $22.57 \pm 2.91$ & $20.81 \pm 1.17$ & 0.19 \\
Implant volume $(\mathrm{mL})$ & $242 \pm 97.76$ & $262 \pm 51.84$ & 0.63 \\
Underlying disease & $\begin{array}{c}\text { Tuberculosis 1 } \\
\text { Hepatitis 1 }\end{array}$ & - & - \\
\hline
\end{tabular}

Values are presented as mean \pm standard deviation. $\mathrm{P}<0.05$ indicates statistical significance. well as partial staining in the implant below the skin and dermal allograft. In one case, a blue-stained necrotic muscle was observed. It was suspected that the injected MBD diffused to the adjacent muscle tissues and the toxicity of the MBD affected the muscle viability. The stained skin margin was biopsied and examined in a histologic examination. Microscopic examinations revealed that necrosis was observed in the dermis and epidermis at the boundary of necrosis along with inflammatory cell infiltration (Fig. 2).

\section{DISCUSSION}

For breast reconstruction, skin-sparing mastectomy or nipplesparing mastectomy allows immediate breast reconstruction using silicone implants. Compared with conventional mastectomy, skin-sparing mastectomy and nipple-sparing mastectomy have many advantages in breast reconstruction with regard to oncological safety [4]. Furthermore, immediate breast reconstruction using silicone implants shortens surgery time with the advantage of greater emotional stability from a faster recovery [6].

In early-stage breast cancer, MBD has been widely used for

\section{Fig. 2. Histologic imaging of necrotic tissue}

Necrosis (black arrows) in the epidermis, dermis, and muscle bundles $\left(H \& E_{1} \times 200\right)$.

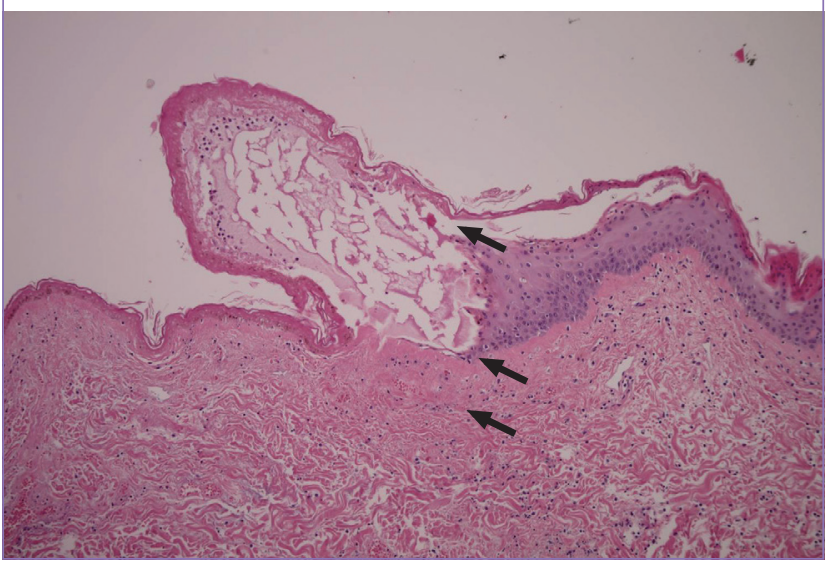

Fig. 1. A case of severe skin necrosis

(A) Severely blue-stained skin with an obvious ischemic lesion. (B) Blue-stained silicone implant. (C) Excised skin (posterior surface).
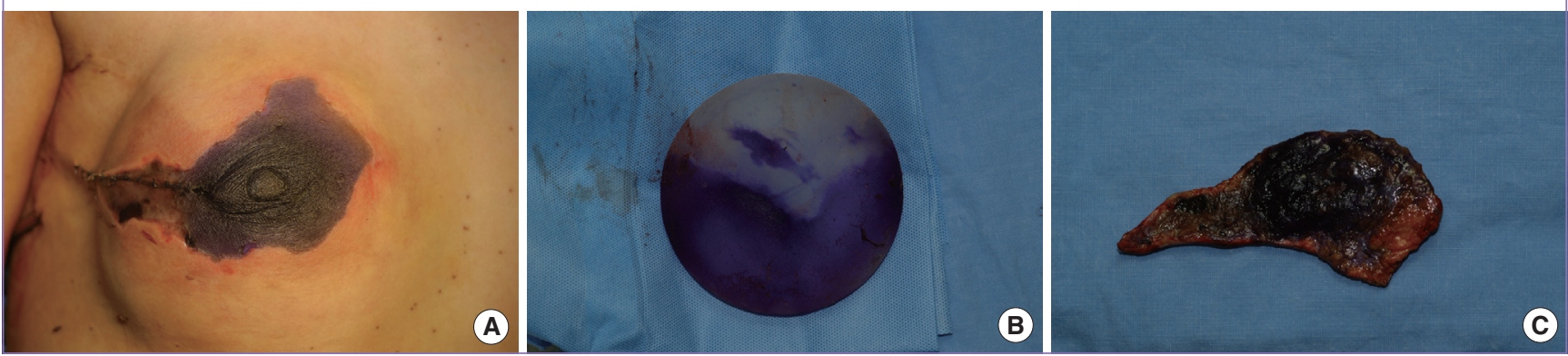
the localization of the sentinel lymph node with a few complications. The complications are reported to occur most often on the remaining skin [7]. Stradling et al. [8] described 5 cases of skin necrosis (21\%) in 24 patients who underwent SLNB using MBD. Complications included erythematous macular lesions, superficial ulcers, and necrotic ulceration at the injection site; the conditions improved with conservative treatment. Zakaria et al. [9] reported complications such as local infection (5\%), skin necrosis (1.25\%), and hypersensitivity of the skin (0.5\%) in 398 patients after the use of MBD. Among five cases of skin necrosis, skin lesions improved with conservative treatment in four. In one case, excision was performed due to the development of full-thickness skin necrosis. Further, Zakaria et al. [9] changed the concentrations of MBD with respect to effectiveness and complications. The success rate of MBD administration in low concentrations displayed no significant difference. Local inflammation (1\%) occurred at the lowest rate in the case of diluted dye (1:7), but no significant difference was found by comparing the dilute concentrations for skin necrosis.

MBD is a cationic thiazine that causes toxic effects to tissues by producing oxides and free radicals, the causes of local inflammation. MBD also has vasoconstrictive effects by inhibiting the nitric oxide-mediated cyclic guanosine monophosphate pathway, which is responsible for smooth muscle relaxation and vasodilation [10]. Therefore, it can be used for refractory septic shock as a rescue therapy. However, after intravenous injection of $1 \% \mathrm{MBD}$ in patients with septic shock, skin necrosis and distal ischemia by extravasation have been reported [10]. Nevertheless, the vasoconstrictive effects of MBD can suppress systemic hypersensitivity, which is a complication of IBD. MBD has also been reported to cause local toxicity, including submucosal ulceration and necrosis of the colon, when it was used as a tattoo marker during laparoscopic colorectal surgery [11].

We experienced six cases of skin necrosis after immediate breast reconstruction after nipple-sparing mastectomy or skinsparing mastectomy with simultaneous SLNB using MBD. In three cases, a wide necrotic area with full-thickness skin necrosis was observed. Excessive blue staining of the skin made it difficult to evaluate the circulation of the NAC and the skin flap. Three days after surgery, the stained skin was observed to have a lower temperature than the rest of the skin, and the desquamation of the skin occurred as a result of bullae formation. When the range of necrosis in the skin was fixed, debridement was planned, and we decided whether to preserve the silicone implant.

There are a number of causes for the necrosis of the mastectomy skin flap. The skin flap thickness and its area can influence mastectomy skin flap necrosis. In our case, most of the mastectomy skin flaps, which were palpated by the surgeon, had consistent thickness among the patients. However, this thickness was difficult to measure during surgery because there is no standardized tool with which to do so. In terms of the skin flap area, it was roughly directly proportional to the implant volume. However, in the complicated cases, there was no relationship between implant sizes and skin necrosis. Therefore, we concluded that MBD was the main cause of necrosis. A histologic examination revealed that necrosis was observed in the dermis and epidermis at the boundary of the stained skin. This finding suggested that the stained skin area corresponded with the necrotic area.

The management methods of necrosis due to $\mathrm{MBD}$ are listed in Table 3. For a small necrotic area, conservative management is sufficient, but if wound dehiscence is found, local skin excision is required. However, in the case of wide and deep skin necrosis, the surgeon should discuss other reconstructive options with the patient. If the patient refuses implant preservation or breast reconstruction, debridement and implant removal should be conducted. If the patient accepts reconstruction, the surgeon should determine whether to salvage the silicone implant. In the case of silicone implant salvage, a latissimus dorsi musculocutaneous flap is considered an appropriate additional breast reconstruction option, which could maintain breast shape and symmetry (Fig. 4) [12]. However, when it is impossible to preserve the implant in a situation such as severe infection, a transverse rectus abdominis myocutaneous (TRAM) flap can be used to

Table 3. Complicated cases of immediate breast reconstruction using methylene blue dye

\begin{tabular}{|c|c|c|c|c|c|c|}
\hline Patient & Age (yr) & Complication & Size $\left(\mathrm{cm}^{2}\right)$ & Additional findings & Treatment & Remarks \\
\hline 1 & 54 & Local inflammation & - & Mildly blue-stained skin & Conservative treatment & \\
\hline 2 & 51 & Tissue necrosis (mild) & $2 \times 1$ & Moderately blue-stained skin and NAC & NAC excision & \\
\hline 3 & 52 & Tissue necrosis (mild) & $1 \times 0.5$ & Moderately blue-stained skin wound dehiscence & Local excision (skin) & \\
\hline 4 & 33 & Tissue necrosis (severe) & $9 \times 4$ & Severely blue-stained skin with implant staining & Pedicled LD flap+implant salvage & Figs. 3,4 \\
\hline 5 & 43 & Tissue necrosis (severe) & $7 \times 5$ & Severely blue-stained skin with implant staining & Silicone implant removal & \\
\hline 6 & 43 & Tissue necrosis (severe) & $9 \times 5$ & $\begin{array}{l}\text { Severely blue-stained skin with implant staining } \\
\text { partial muscle necrosis }\end{array}$ & Silicone implant removal & Fig. 1 \\
\hline
\end{tabular}


Fig. 3. A case of blue-stained skin necrosis

(A) Severely blue-stained thin skin with necrotic changes. (B) Excised skin (posterior surface).
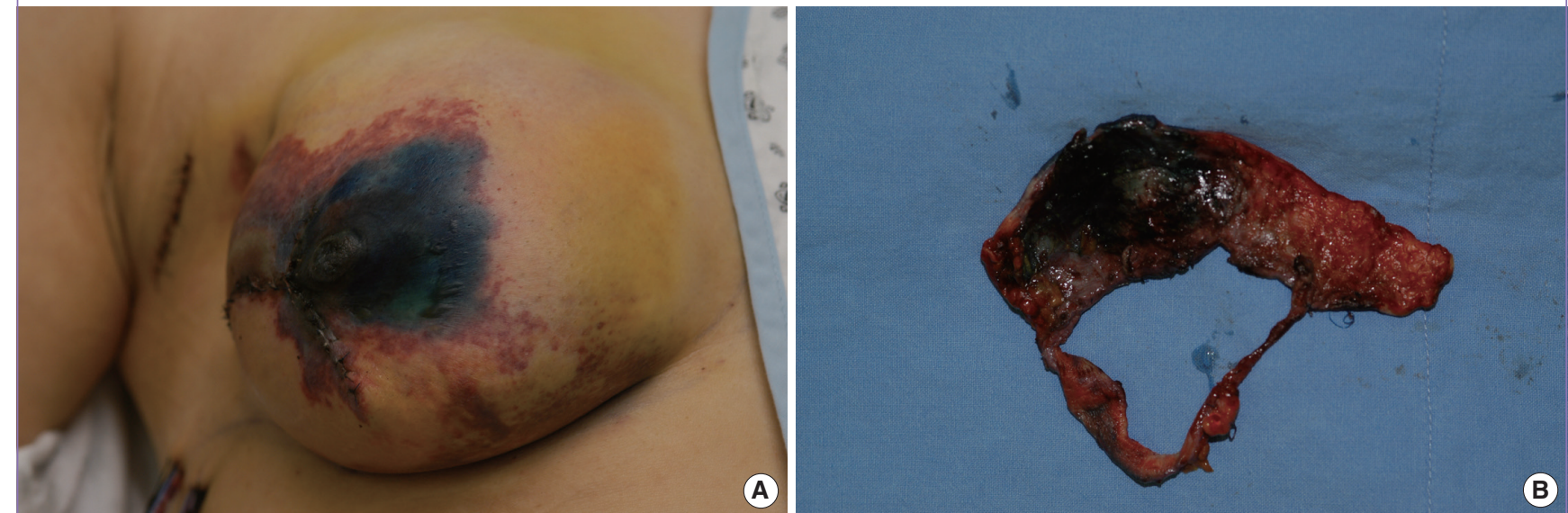

Fig. 4. Postoperative results after breast reconstruction

(A-D) A patient reconstructed with a latissimus dorsi musculocutaneous flap (6 months later).
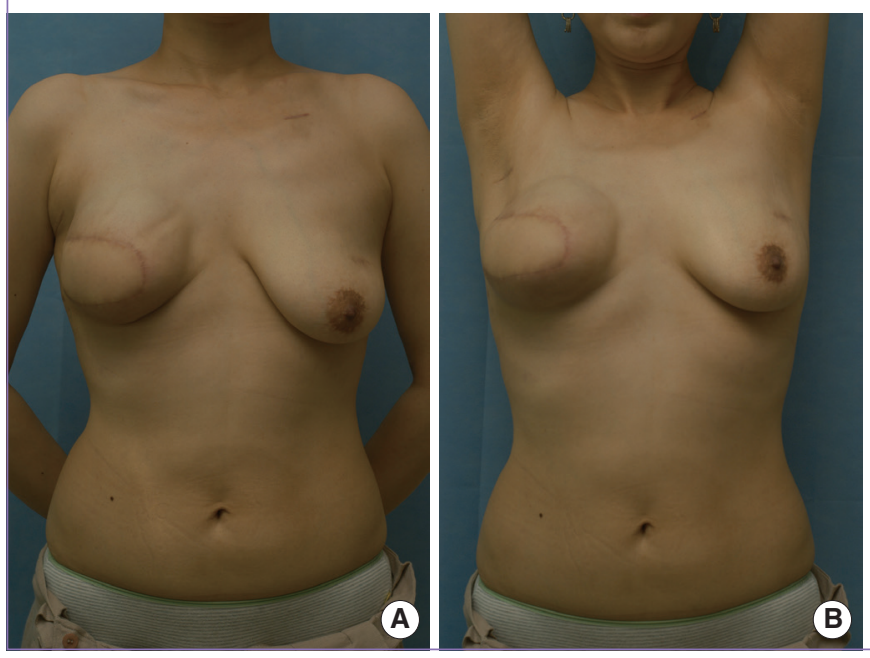

produce satisfactory results.

When the breast surgeon performed nipple-sparing mastectomy or skin-sparing mastectomy using MBD in the same manner as with modified radical mastectomy (MRM), we observed more complications in the case of immediate breast reconstruction than in the case of only modified radical mastectomy. There are several possible causes. First, the breast skin flap of skin-sparing mastectomy is wider than that of MRM. Second, the spared NAC demands a great deal of blood supply. Third, breast implantation caused more tension on the skin flap, which disturbed the blood flow. Finally, blue-stained skin makes it difficult to evaluate the skin envelope and to make a decision on whether reconstructive surgery is possible.

There are additional factors that may aggravate the condition. A ptotic breast has a wider skin flap than does a non-ptotic breast with the same volume. Sometimes, thin skin may cause

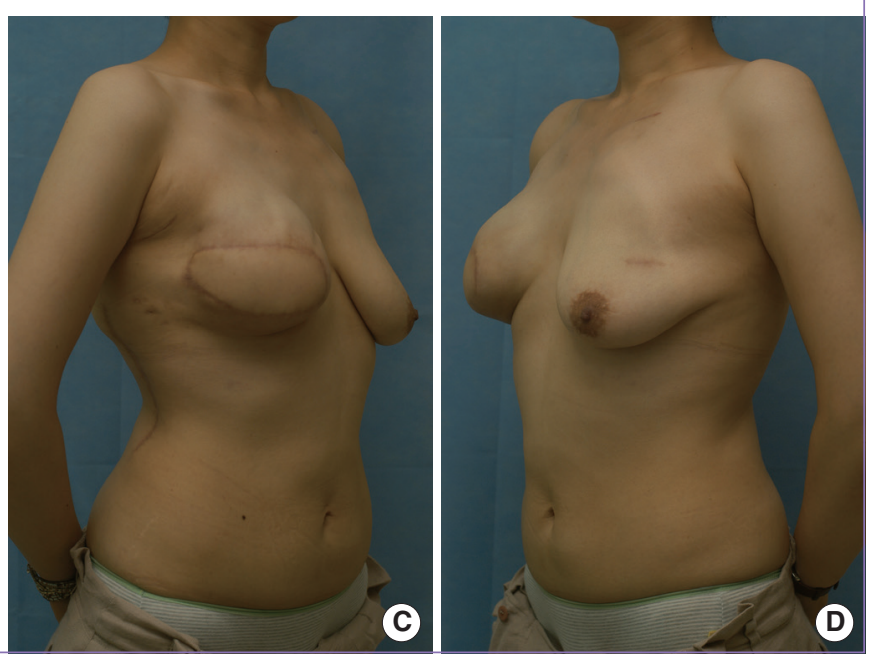

trouble. Thin skin may either be innate or caused by weight control. Further, when the breast mass is located near the skin, surgeons are compelled not to preserve a sufficient thickness of the skin. If the remaining skin is very thin with little subcutaneous tissue, it becomes vulnerable to hemodynamic stress and prone to postoperative skin necrosis. Therefore, at preoperative evaluation, if a patient has a ptotic breast or thin skin, the surgeon should consider the fact that the use of silicone implants may lead to unfavorable results with complications. In such cases, TRAM flap reconstruction can be an alternative method for satisfactory outcomes.

However, most importantly, we think that the use of MBD should be discussed in the preoperative period between breast surgeons and plastic surgeons. Moreover, for breast reconstruction, breast surgeons should develop a safer protocol for the use of MBD. This is likely to require the reduction of both the 
injection volume (approximately 2-3 mL) and the concentration of MBD. Furthermore, the location of the injection must be considered. To prevent excessive skin staining, it is preferable to inject MBD into the peritumoral or intratumoral area. When breast surgeons are using MBD, doctors should give patients a detailed explanation of the management and potential complications of MBD, such as skin necrosis.

\section{REFERENCES}

1. American Society of Breast Surgeons. Consensus statement on guidelines for performing sentinel lymph node dissection in breast cancer [Internet]. Columbia: American Society of Breast Surgeons; c2010 [cited 2005 Dec 8]. Available from: https://www. breastsurgeons.org/statements/PDF Statements/SLN_Dissection.pdf.

2. Nagao T, Kinoshita T, Hojo T, et al. Sentinel lymph node biopsy using indigo carmine blue dye and the validity of ' $10 \%$ rule' and ' 4 nodes rule.' Breast 2012;21:455-8.

3. Simmons RM, Smith SM, Osborne MP. Methylene blue dye as an alternative to isosulfan blue dye for sentinel lymph node localization. Breast J 2001;7:181-3.

4. Kinoshita S, Nojima K, Takeishi M, et al. Retrospective comparison of non-skin-sparing mastectomy and skinsparing mastectomy with immediate breast reconstruction. Int J Surg Oncol 2011;2011:876520.

5. Niemeyer M, Paepke S, Schmid R, et al. Extended indica- tions for nipple-sparing mastectomy. Breast J 2011;17:296-9.

6. Cho YK, Yang JD, Kim GR, et al. Immediate implant reconstruction using silicone prosthesis in breast cancer patients after skin sparing mastectomy. J Korean Soc Plast Reconstr Surg 2010;37:749-57.

7. Reyes F, Noelck M, Valentino C, et al. Complications of methylene blue dye in breast surgery: case reports and review of the literature. J Cancer 2010;2:20-5.

8. Stradling B, Aranha G, Gabram S. Adverse skin lesions after methylene blue injections for sentinel lymph node localization. Am J Surg 2002;184:350-2.

9. Zakaria S, Hoskin TL, Degnim AC. Safety and technical success of methylene blue dye for lymphatic mapping in breast cancer. Am J Surg 2008;196:228-33.

10. Dumbarton TC, Gorman SK, Minor S, et al. Local cutaneous necrosis secondary to a prolonged peripheral infusion of methylene blue in vasodilatory shock. Ann Pharmacother 2012;46:e6.

11. Lane KL, Vallera R, Washington K, et al. Endoscopic tattoo agents in the colon. Tissue responses and clinical implications. Am J Surg Pathol 1996;20:1266-70.

12. Mosahebi A, Ramakrishnan V, Gittos M, et al. Aesthetic outcome of different techniques of reconstruction following nipple-areola-preserving envelope mastectomy with immediate reconstruction. Plast Reconstr Surg 2007;119:796803. 